in the book, for Lorenz founds ethology in the philosophy of Gestalt psychology, in physiology and in embryology. These three fill the main sections of the book: one on philosophy and methods, and two marked by Lorenz's controversial distinction between innate and acquired information. He repeats once again his argument for a fundamental distinction between innate and acquired information, and, in the present environment of debates about "genetic determinism", it is undoubtedly worth repeating. The fact that the behaviour of animals is adapted to their environments means that they must have "information" about their environment; it is this information (not behaviour) which Lorenz dichotomizes into genetic and acquired.

Lorenz could have written a book with the conceptual content of Foundations of Ethology 30 years ago. If it had been published then it would have become a classic alongside Tinbergen's Study of Instinct (Clarendon, 1951). Coming out now it appears awkwardly antediluvian. In his foreword, T.H. Bullock says that the book is intended as a contribution to the history of ethology. But to write a period textbook is not to write the history of a period. Foundations of Ethology, however, is not even simply a period textbook, for Lorenz is far too concerned with defending his concepts against later criticism. The fault with the book can be

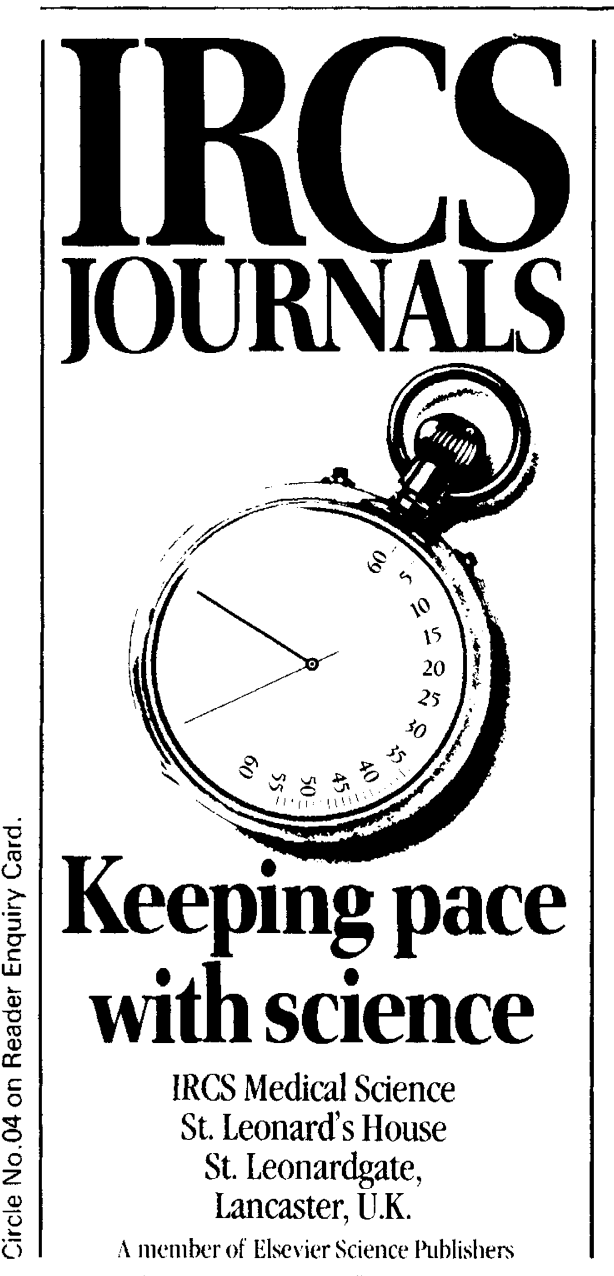

clarified by an analogy with historiography. By tracing back the pedigree of ideas that are interesting now, it is easy to distort history, because isolated ancestral ideas are rarely representative of contemporary thinking. Lorenz, by tracing forward and defending his ideas, produces an extraordinary futuristic distortion. For example, in his discussion of motivation he defends himself against Robert Hinde's criticisms and even presents an updated hydraulic analogy. But modern work on motivation is established on quite separate foundations, manifesting itself to most of us only as a fascinating and imposing castle, with sheer walls of solid algebra, surrounded by minefields of exegesis.

Lorenz's later books have tended to be increasingly concerned with human beings, but this one is almost entirely about animal

\section{Galactic sociology}

\section{John D. Barrow}

Galactic Astronomy: Structure and Kinematics, 2nd Edn. By Dimitri Mihalas and James Binney. Pp.597. ISBN 0-7167-1280-6. (W.H. Freeman: 1981.) $\$ 29.95, £ 20.70$.

THE story is told of a lecturer beginning his address on the structure of stars with the words "Stars appear to be very simple objects", whereupon someone in the audience retorted "You'd look pretty simple too at a distance of a hundred light years!". Because they are a good deal farther away than stars, astronomers used to think that galaxies were even simpler. Until very recently elliptical galaxies were thought to be uncomplicated and understood - just isothermal "gases" of stars whose various degrees of ellipticity were merely reflections of their differing rotation rates. During the past six years this naive picture has been obliterated and replaced by that complexity, masquerading as simplicity, that is the hallmark of all natural phenomena. Ellipticals have been revealed as complicated systems with threedimensional dynamics that are neither isothermal nor rotating to any significant degree. Their flattening is a consequence of much more subtle dynamical properties.

Surprisingly, there has never been an authoritative advanced textbook on the structure of galaxies. There are indeed several good introductory texts and collections of review articles, either scattered around the literature or gathered into massive review volumes that send a shiver down your cheque book, but no single, detailed and up-to-date guide to all the relevant observational data and theoretical ideas - until now.

Mihalas and Binney have completed the first part of a two-volume treatise on the subject, this book being confined to obser- behaviour. There is a typically nonconciliatory appendix on the uniqueness of mankind and a stricture or two against the deadly sins of civilized man. Our "innate releasing mechanisms", we are told on p.166, give us an unhealthy liking for chocolate and "even the most complete insight into the workings of our IRMs does not make it easy to avoid suicide by overeating". As a gastronomic experience, Foundations of Ethology is not so much suicidal as dry and unappetizing; it rather resembles three full courses of stale bread. Well before I had reached p.166, I had found that it went down best when taken with religeuses and éclairs au chocolat.

Mark Ridley is a Junior Research Fellow of Oriel College, and a member of the Animal Behaviour Research Group, University of Oxford.

vations and kinematics. The second volume, to be written by Binney and Tremaine, will be devoted to dynamics. By any standards this first volume is outstanding. Although advertised as a second edition of Mihalas and Routly's book of the same title, do not be misled, this is an entirely new book of more than double the length and considerably more depth than its prototype. After a brief history and introduction to astronomical concepts and vocabulary, it begins with a detailed overview of stellar evolution and the interstellar medium before describing the stellar content and kinematics of external galaxies and the Milky Way. The final chapters provide studies of the hydrogenic content of galaxies and their rotational properties. References to the original literature are always provided, although through a rather cumbersome system, and good tables and diagrams illuminate the text throughout.

This book is essential for any graduate course on galaxies. It is too advanced for undergraduate courses although lecturers and more able students will want to refer to it. Most graduate courses on galaxies divide their time equally between the material covered in this volume and the dynamical theory that will appear in Vol. 2, and together the two books should provide everything such students require. Unfortunately, it is hard to believe that many of them will be willing or able to pay the considerable price of both volumes - one hopes that a paperback edition will soon be available, as all astronomers should own and read this book. Perhaps also the authors and publishers might consider a third volume which would be an abridged combination of the two earlier volumes, with a length and level comparable to the original edition written by Mihalas and Routly.

John Barrow is a Lecturer in Astronomy at the University of Sussex. 